\title{
Wachstumsmodelle und Krisenmuster in Osteuropa
}

Mit Blick auf die Stabilität des Euro-Raumes richtet sich das öffentliche Interesse momentan vor allem auf die südeuropäischen Krisenländer. Allerdings waren einige osteuropäische Länder noch stärker von der Krise betroffen als Griechenland oder Portugal. Das gilt speziell für die baltischen Länder. Generell war hier die Krisenbetroffenheit in den Ländern mit verschuldungsgetriebenen Wachstum und besonders hohen Handelsbilanzdefiziten besonders ausgeprägt. Günstiger schnitten die osteuropäischen Länder mit stärkerer Exportorientierung ab. Die Anti-Krisen-Politik nahm in Osteuropa vielfach die Muster der extremen Sparpolitik in Griechenland und Portugal vorweg - und zeitigte sehr problematische Resultate.

\section{Einleitung}

Die jüngste Finanz-und Wirtschaftskrise hat in Osteuropa sehr unterschiedliche Wirkungen gezeigt. Polen ist bislang nicht in die Rezession gerutscht, dagegen gehören die baltischen Länder, aber auch die Ukraine, weltweit zu den Ökonomien mit der stärksten Krisenbetroffenheit. Sucht man nach den Gründen für derart unterschiedliche Krisenverläufe, so fällt zunächst auf, dass das polnische Wachstumsmodell relativ stark von Exporten getragen wird, während für die vier besonders schwer von der Krise betroffenen Länder der finanzgetriebene Charakter der Akkumulation in den Jahren vor der Krise charakteristisch ist. Vor diesem Hintergrund ist es eine Zielstellung dieses Beitrages, der Frage nachzugehen, wie die Krisenverläufe in Osteuropa mit den jeweiligen Akkumulationsmodellen zusammenhängen. Hierbei lassen sich grundsätzlich zwei Modelle in Osteuropa unterscheiden: ein vornehmlich exportorientiertes Modell und ein finanziarisiertes Modell, das sich sehr stark auf Kapitalimporte und einen Immobilienboom stützte. Die Klärung des Zusammenhanges zwischen den unterschiedlichen Wachstumsmodellen und Krisenverläufen ist keineswegs nur von wissenschaftlichem, sondern auch von praktischem Interesse: Denn das jeweilige Wachstumsmodell hat auch Konsequenzen für die Optionen einer Anti-Krisen-Politik. Auch diesen soll in dem Beitrag nachgegangen werden. Hierbei ist zudem von Interesse, ob es einen Unterschied macht, ob ein Land EUMitglied ist oder nicht. Aus dieser Analyse wird sich eine Landkarte der Krisenmuster und Anti-Krisen-Politiken ergeben.

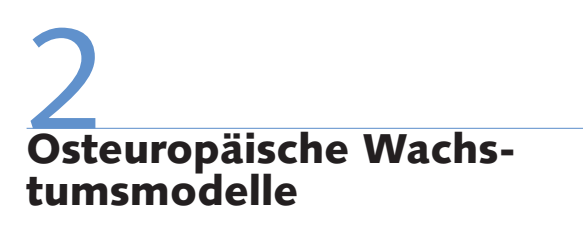

Generell sind für die osteuropäischen Transformationsökonomien eine starke Außenorientierung der Entwicklung und eine Dominanz transnationaler Konzerne in den wirtschaftlichen Schlüsselsektoren, vor allem im Finanz-, oft aber auch im Industriesektor, charakteristisch. Diese Tendenzen sind durch die Europäische Kommission in den Beitrittsverhandlungen stark forciert worden. Daher sind sie in den neuen Mitgliedsländern, aber auch in faktischen Kandidatenländern Südosteuropas, noch stärker ausgeprägt als in den sowjetischen Nachfolgestaaten, von denen hier Weißrussland und die Ukraine in die Analyse einbezogen werden.

In der jeweiligen Art der Außenorientierung unterscheiden sich die osteuropäischen Länder hingegen gravierend. In den Visegrád-Staaten (Polen, Slowakei, Tschechische Republik, Ungarn), in Slowenien und Weißrussland ist zum einen eine starke Prägung durch den industriellen Export zu erkennen. Zum anderen wurde das Wachstum in den Vorkrisenjahren zugleich aber auch durch zunehmende Verschuldung getragen. Insofern hat dieses Wachstumsmodell auch Züge einer Finanziarisierung. In den baltischen und südosteuropäischen Ländern, aber auch in der Ukraine, waren die Akkumulationsmodelle primär als finanziarisiert zu charakterisieren. Ein sehr rasches Kreditwachstum war der zentrale Wachstumsmotor. Dieses wurde durch hohe Kapitalzuflüsse genährt. Überbewertete Wechselkurse stützten die Verschuldungsdynamik und führten gleichzeitig zu einem Importsog. Die Handels- und Leistungsbilanzen waren tief in den roten Zahlen. Dieses Akkumulationsmodell lässt sich als abhängige Finanziarisierung kennzeichnen (vgl. Becker 2010, S. 523ff.; Myant/Drahokoupil 2011, S. 299ff.).

\section{Krisendynamiken im ex- portorientierten Modell}

Das Akkumulationsmodell in den Visegrád-Ländern, in Slowenien, aber auch in Weißrussland ist eindeutig durch den industriellen Export getragen. Die VisegrádLänder und Slowenien sind seit den 1990er Jahren sehr stark über Produktionsketten an die deutsche Exportindustrie gebunden worden (Mazzocchi 2010, S. 261). Dagegen orientiert sich der weißrussische Export immer noch sehr auf den russischen Nachbarn, der mit einem Anteil von 36,6\% auch 2007 nach wie vor der wichtigste äußere Absatzmarkt war (Marzęda 2009, S. 152, Tab. 10). In den Visegrád-Ländern gewannen transnationale Konzerne in der Transformationsphase eine dominante Position in der industriellen Exportproduktion und forcierten erhebliche Struk-

\footnotetext{
Joachim Becker, Dr., ist a.o. Professor am Institut für Außenwirtschaft und Entwicklung der Wirtschaftsuniversität Wien. Arbeitsschwerpunkte: Entwicklungsökonomie, Regulations- und Staatstheorie, Regionale Integration, Krisen. e-mail: joachim.becker@wu.ac.at
} 
turveränderungen in der Industrie. Die Auslandsunternehmen wurden von relativ niedrigen Löhnen bei einer gleichzeitig recht hohen Qualifikation angezogen. Ihre osteuropäischen Betriebe haben oftmals eine Zulieferfunktion; Forschungs- und Entwicklungsaktivitäten finden in der Region kaum statt. In den kleinen Ländern ist das Produktionsprofil oft sehr eng. In der Slowakei beispielsweise machte der Autoexport 2007 etwa $40 \%$ der Gesamtausfuhr aus (Okáli et al. 2008, S. 52). In Slowenien, vor allem aber in Weißrussland, war die Relevanz einheimischer Unternehmen höher. Slowenische Klein- und Mittelbetriebe hatten bereits zu jugoslawischen Zeiten eine starke Westorientierung, während die weißrussische Industrie in der sowjetischen Arbeitsteilung eine zentrale Rolle im Maschinen- bzw. auch im Traktorenbau spielte. Beide Spezialisierungsmuster haben bis heute eine gewisse Relevanz.

Trotz der Exportorientierung waren die Handelsbilanzen dieser Länder - mit Ausnahme der Tschechischen Republik im Regelfall negativ. Noch stärker fielen für die negativen Leistungsbilanzen allerdings die hohen Gewinnrepatriierungen westlicher Unternehmen ins Gewicht. 2008 belief sich das entsprechende Defizit in der Einkommensbilanz in der Tschechischen Republik und Ungarn bereits auf ca. $8 \%$ des Bruttoinlandsproduktes (BIP). Die Leistungsbilanzdefizite bewegten sich meist um die als kritisch geltende Grenze von $5 \%$ des BIP herum (Astrov/Pöschl 2009, S. 357, Tab. 7).

Die relativ niedrigen Löhne dämpften die Binnenmarktentwicklung. In den letzten Jahren vor der Krise wurde der Kauf von Konsumgütern und Wohnraum zunehmend von Krediten angeheizt. In Metropolen wie Bratislava, Prag oder Warschau stiegen die Wohnungspreise sehr rasch, die Entstehung von Immobilienblasen war erkennbar. Die Verschuldung erfolgte allerdings - mit Ausnahme Ungarns überwiegend in einheimischer Währung. Besonders rasch war das Kreditwachstum in Slowenien, das den Euro 2008 einführte (Becker 2010, S. 518). Auch in Weißrussland mit seiner stark staatlich gelenkten Ökonomie und seinem überwiegend staatlichen Bankensektor stieg das Kreditvolumen zwischen 2003 und 2006 von etwa 15 auf $25 \%$ des BIP (Barisitz 2009, S. 227). Zwischen dem Wachstum des BIP und dem der Kredite bestand in der Tendenz eine enge Korrelation (Dutrénot/Sand-Zantman
2010, S. 167). Als zentrale Verwundbarkeiten des Akkumulationsmodells waren die starke Exportabhängigkeit und die starke Kreditexpansion erkennbar.

Die Ökonomien der primär exportorientierten Ländergruppe waren am deutlichsten vom starken Einbruch der Exporte betroffen. Für die osteuropäischen Mitgliedsländer war dies Folge des markanten Rückgangs der deutschen Exportproduktion, für Weißrussland resultierte der Einbruch aus dem starken Rückgang des BIP im benachbarten Russland. Während der Exporteinbruch im 4. Quartal 2008 vielfach noch milde war, erreichte er im 1. Quartal 2009 in den Visegrád-Ländern und Slowenien zwischen etwa $25 \%$ und $30 \%$ gegenüber dem Vorjahr (Astrov/ Pöschl 2009, S. 348, Tab. 1).

Im Verlauf des Jahres 2009 schwächte sich der Rückgang etwas ab, sodass die Exporte der Visegrád-Länder und Sloweniens 2009 insgesamt knapp ein Fünftel niedriger lagen als 2008 (Pöschl 2010, S. 432, Tab. 4). Als besonders nachteilig erwies sich die enge Spezialisierung der kleinen Ökonomien auf den Automobilsektor, der in der Krise einen besonders schweren Einbruch erlitt. Der Exportrückgang Polens mit seiner breiter gefächerten Exportwirtschaft und geringen Dominanz des transnationalen Kapitals fiel etwas schwächer aus als in den anderen Ländern der Region. Für Polen und die Tschechische Republik erwies sich 2009 als günstig, dass ihre Währungen - im Gegensatz zur Slowakei und Slowenien - abwerteten (Workie et al. 2009, S. 96, 101).

2010 erholten sich die Exporte und folglich auch die Industrieproduktion. Die enge Bindung der exportorientierten Ökonomien der neuen EU-Mitgliedsländer blieb bestehen - und damit auch die Verwundbarkeit gegenüber dem Auf und $\mathrm{Ab}$ der deutschen Exportkonjunktur. Diese Verwundbarkeit wurde während der ersten Zuspitzung der Krise nur sehr vereinzelt kritisch kommentiert, beispielsweise durch den slowenischen Staatspräsidenten Danilo Türk in einem Interview mit dem serbischen Nachrichtenmagazin NIN (Murahemi-Vukas 2009, S. 67). Mit der Erholung der deutschen Exportwirtschaft waren die Schattenseiten der Exportspezialisierung und engen Bindung an die deutsche Ökonomie dann kein Thema mehr. Der deutsche Exportaufschwung ist jedoch angesichts der Krise der Euro-Zone und der Austeritätspolitik in vielen EU-Ländern, speziell der europäischen Peripherie, als instabil einzuschätzen. Für Weißrussland erodieren aus politischen Gründen günstige Sonderbedingungen im Handel mit Russland, was das weißrussische Wachstumsmodell zumindest beeinträchtigen wird (Kłysiński 2011, S. VII).

Von den Turbulenzen auf den Finanzmärkten war zunächst die privatisierte Alterssicherung betroffen (Morvay et al. 2010, S. 6). Restriktivere Refinanzierungsbedingungen und eine striktere Kreditvergabepolitik wurden erst später fühlbar. Dies schlug vor allem auf die Immobilienund Bauwirtschaft durch. Hier waren die Wirkungen in Slowenien wegen der stark erhöhten Abhängigkeit von externer Refinanzierung und des starken Immobilienbooms besonders stark (Marn 2010; Urbinati 2010, S. 331). Die Verschuldung der Privathaushalte setzte sich auch während der Krise weiter fort (Schreiner et al. 2010, S. 15). Sie stabilisierte zwar den Konsum ein Stück weit; gleichzeitig wurde damit aber auch ein finanziarisiertes Akkumulationsmuster fortgeschrieben. Es ist auch für die Visegrád-Länder und Slowenien erkennbar, dass die private Verschuldung zu einer Achillesferse des Modells wird. Auch der Bankensektor in Weißrussland geriet unter Druck, die Abschwächung der Dynamik der Kreditvergabe war allerdings schwächer als in anderen Nachfolgestaaten der Sowjetunion in der Region (Barisitz et al. 2010, S. 51, Abb. 2).

Unter den primär exportorientierten Ökonomien war 2009 der BIP-Rückgang in den beiden kleinen Euro-Ländern Slowenien und Slowakei mit 7,8 und 4,7\% am stärksten. Die Tschechische Republik folgte mit einem Rückgang von 4,2\% (Pöschl 2010, S. 428, Tab. 1). Die weißrussische Ökonomie stagnierte (Barisitz et al. 2010, S. 51, Tab. 1), während die polnische Ökonomie um 1,7 \% wuchs (Pöschl 2010, S. 428, Tab. 1).

Die bereits erwähnte wirtschaftliche Erholung ab 2010 in allen Ländern dieser Gruppe fiel in Slowenien mit der ausgeprägteren Finanzkrise am schwächsten aus (Schreiner et al. 2010, S. 9, Abb. 2). Mit Ausnahme der Slowakei sind die Arbeitslosenraten in den exportorientierten Visegrád-Ländern sowie Slowenien trotz der wirtschaftlichen Erholung in der Tendenz weiter gestiegen (Schreiner et al. 2010, S. 13, Abb. 5). In der Slowakei lag sie in dieser Ländergruppe mit 14,5\% im Spitzenfeld, die Jugendarbeitslosigkeit war 
mit einer Quote von 36,6 \% im November 2010 sogar die zweithöchste in der EU überhaupt (Eurostat 2011a). In Polen blieb die Arbeitslosigkeit mit offiziell ca. $10 \%$ hoch, wobei es außerdem noch eine substanzielle verdeckte Unterbeschäftigung in der Kleinlandwirtschaft gibt. Auffällig ist, dass die beiden größeren, etwas stärker binnenorientierten und diversifizierten Ökonomien Polens und Weißrusslands bislang besser durch die Krise gekommen sind als die kleinen, eng spezialisierten Exportökonomien. In beiden Ländern spielte die Binnennachfrage bei der Stabilisierung bzw. dem Wiederaufschwung eine verhältnismäßig wichtige Rolle. Für Weißrussland haben sich jedoch im Frühjahr 2011 aufgrund der schärferen Gangart der russischen Regierung und der aufgrund der scharfen Repression nach den Wahlen erfolgten politischen Isolierung die außenwirtschaftlichen Bedingungen so weit geändert, dass es zu einer erkennbaren Devisenknappheit gekommen ist (Ludwig 2011, S. 3).

\section{4 \\ Krisendynamiken im finanziarisierten Modell}

Die südosteuropäischen und baltischen Länder sowie die Ukraine wiesen ein primär finanziarisiertes Akkumulationsregime auf. Auch Ungarn zeigte sehr ausgeprägte Muster der abhängigen Finanziarisierung, war allerdings deutlich stärker exportorientiert als die anderen Länder dieser Gruppe.

\subsection{VERSCHULDUNGSPROBLEME}

Die wirtschaftliche Dynamik der Vorkrisenjahre war in dieser Ländergruppe primär durch eine rapide steigende Verschuldung gekennzeichnet. Zwischen 2003 und 2006 verdoppelte sich der im Inland an den Privatsektor vergebene Kredit in den südosteuropäischen und baltischen Ländern in etwa und erreichte in Lettland und Estland 2006 bereits fast $80 \%$ des BIP (Frangakis 2009, S. 64, Tab. 3.8). Die Kredite alimentierten Immobilienbooms und den Konsum der Mittelschicht. Deren Einkommen war eigentlich nicht ausreichend, um das angestrebte Konsumniveau zu erreichen. Die Bindung an die neoliberale Wirtschaftspolitik erfolg- te stark über den verschuldeten Konsum. Es entstand eine verschuldungsbasierte Konsumgesellschaft (vgl. Burić 2010). Im Gegensatz etwa zur Tschechischen Republik und der Slowakei wurden die Kredite überwiegend in Fremdwährung aufgenommen, da für Fremdwährungskredite niedrigere Zinsen zu zahlen waren. In den baltischen Ländern erreichten die Devisenkredite einen Anteil von etwa $80 \%$ (Astrov/Pöschl 2009, S. 356, Abb. 5). Die im westlichen Eigentum stehenden Banken hatten ihrerseits ein Interesse, Kredite in den Währungen ihrer Herkunftsländer $\mathrm{zu}$ vergeben. Besonders aggressiv in der Vergabe von Devisenkrediten waren die Töchter österreichischer Banken. Laut dem Finanzmarktstabilitätsbericht der Oesterreichischen Nationalbank (OeNB) vom Dezember 2010 entfielen im 4. Quartal 2009 auf die Töchter österreichischer Banken in Kroatien, Ungarn, Rumänien, Russland und der Ukraine $71 \%$ der Devisenkredite (Pann et al. 2010, S. 65), was deutlich über deren Marktanteil lag. Die Devisenverschuldung implizierte eine besondere finanzielle Verwundbarkeit. Jede Abwertung der nationalen Währung hatte absehbar große Probleme der Fremdwährungsschuldner zur Folge, da diese Einkommen in nationaler Währung bezogen, aber in Devisen verschuldet waren. Eine Währungsabwertung bedeutete für sie absehbar eine höhere Schuldenlast. Damit war die Mittelschicht eng an die Wechselkurspolitik gebunden, die ein zentraler Bestandteil der neoliberalen Wirtschaftspolitik war.

Die starke Kreditexpansion und die Immobilienblasen wurden durch die Kapitalzuflüsse und die damit einhergehende rapide steigende Auslandsverschuldung alimentiert. Diese war nicht allein in den neuen EU-Ländern zu beobachten, sondern auch in den Nachfolgestaaten Jugoslawiens und der Ukraine. In der Ukraine war - ähnlich wie in Russland im letzten Jahrzehnt - eine extrem rasche Zunahme der Auslandsverschuldung des privaten Unternehmenssektors und speziell der Banken zu konstatieren. So stieg zwischen dem 1.1.2004 und dem 1.1.2009 die Auslandsverschuldung der ukrainischen Banken von 1,9 Mrd. US\$ auf 39,4 Mrd. US\$, jene des sonstigen Privatsektors von 10,8 Mrd. US\$ auf 38,7 Mrd. US\$ (Petković/ Nikolić 2009, Tab. 5.10). Die Kredite hatten meist nur eine Laufzeit von ein bis drei Jahren (Žold’ 2010, S. 44). Vor allem die hoch im Ausland verschuldeten Länder wiesen eine ungünstige Schuldenstruktur auf und waren damit besonders verwundbar (vgl. Astrov/Pöschl 2009, S. 355, Tab. und S. 356, Abb. 6).

\subsection{BEDEUTUNG DER WECHSELKURSE}

Wechselkurse mit nominaler oder zumindest realer Aufwertungstendenz waren die zentrale wirtschaftspolitische Grundlage der kreditgetriebenen Akkumulation. Ein Teil der Länder hatte fixe Wechselkursregime - teils sogar mit einem Currency Board und einer damit verbundenen Bindung der Geldmenge an den Devisenbestand, ein anderer Teil beförderte durch eine Hochzinspolitik die Aufwertung (Becker 2010, S. 523). Offiziell begründet wurde die Einführung derartiger Wechselkursregime und einer Hochzinspolitik mit der Inflationsbekämpfung. Sie galt als stabilitätsorientiert. Tatsächlich schuf sie aber die Grundlagen für Instabilität. Die Überbewertung der nationalen Währungen schwächte über die Verbilligung der Importe die schwachen nationalen Produktionsstrukturen noch weiter. Die Handels- und Leistungsbilanzdefizite waren exorbitant - und das galt auch für die äußere Verschuldung, mit der diese Defizite finanziert wurden. Die Leistungsbilanzdefizite lagen - außer in der Ukraine, die eine recht starke Schwerindustrie im Osten des Landes aufweist, und dem stärker exportorientierten Ungarn - deutlich jenseits der kritischen Grenze von $5 \%$ des BIP. In Lettland und Bulgarien übertrafen sie in den Vorkrisenjahren die Marke von $20 \%$ des BIP, und in Montenegro wurden 2008 sogar 32,6\% des BIP erreicht (Becker 2010, S. 524). Die mit dieser Konstellation verbundene extreme Krisenanfälligkeit wurde in den meisten Ländern ignoriert. Am ehesten waren kritische Stimmen noch in Kroatien und Serbien zu hören, wo die Erinnerungen an die Verschuldungskrise Jugoslawiens in den 1980er Jahren noch nicht verblasst sind. So warnte eine Gruppe von kroatischen Ökonomen bereits 2001 vor den Gefahren, die mit einer teilweisen Euroisierung der kroatischen Ökonomie, wie sie vom Internationalen Währungsfonds (IWF) damals forciert wurde, verbunden waren (Baletić et al. 2010, S. 40ff.). Der serbische Ökonom Mladen Kovačević (2009, S. 265, 271) wies auf einem Seminar kurz vor der Zuspitzung der internationalen Krise auf strukturelle Ähnlichkeiten der Krisenverwundbarkeit 
Serbiens mit Russland unmittelbar vor der Krise Ende der 1990er Jahre hin.

Die Krise lief in den primär finanziarisierten Ökonomien vorrangig über den Finanzkanal ab (Becker 2010, S. 525 ff.; Drezgić 2010, S. 207). Rückläufige Kapitalzuflüsse bzw. Kapitalabflüsse entzogen dem finanziarisierten Modell die Grundlage. Immobilienboom und Konsumnachfrage brachen - z.T. scharf - ein. Währungsabwertungen standen auf der Tagesordnung. In Staaten mit sehr rigidem Wechselkursregime - wie den baltischen Ländern, Bulgarien, Kroatien und Bosnien-Herzegowina - versuchten die Regierungen die Währungsparität um jeden Preis zu halten, da sie fürchteten, dass bei einer Abwertung die Devisenschuldner und die Banken unmittelbar unter Druck geraten würden. Sie leiteten scharfe Sparprogramme - teils in Zusammenspiel mit dem IWF und Europäischer Kommission, teils in Eigenregie - ein. Diese vertieften die Rezession (Becker 2010, S. 525). In mehreren Ländern mit flexiblen Wechselkurs - vor allem Ungarn, der Ukraine, Rumänien, Serbien - setzte im Herbst 2008 eine Abwertung ein, die besonders in Ungarn und der Ukraine aufgrund der hohen Devisenverschuldung das Bankensystem unter starken Druck setzte (Becker 2009, S. 101f.). In der Ukraine, aber auch in den südosteuropäischen Ländern (vor allem Serbien und Kroatien), zogen Anleger Kapital aus den Banken ab. Insbesondere in den Ländern mit frischen Erinnerungen an die Finanzkrisen in den 1990er Jahren - Serbien, Kroatien, Bosnien-Herzegowina und Albanien - erfolgte die Wiederanlage des Geldes trotz vertrauensbildender Maßnahmen der Regierungen eher schleppend (Dvorsky et al. 2010, S. 82). In den Ländern mit sehr starker Rezession - wie den Baltischen Ländern, der Ukraine und Rumänien - gerieten die Schuldner und damit auch die Banken durch die schlechte wirtschaftliche Lage unter Druck. Besonders stark kumulierte die Wirkung von hoher Devisenverschuldung, Abwertung und scharfer Rezession in der Ukraine und in Rumänien. In der Ukraine erreichte der Anteil notleidender Kredite im 2. Quartal 2010 40,2 \%, in Rumänien 26,2 \% (OeNB 2010, S. 24, Abb. 13). In beiden Ländern waren österreichische Banken sehr stark engagiert. Von der tiefen Krise der baltischen Länder waren vor allem die schwedischen Banken betroffen.

Die restriktivere Kreditvergabepolitik und die Austeritätspolitik ließen die Bin- nennachfrage und die Investitionen stark einbrechen. Außerdem gingen die Exporte zurück. Die Ukraine war aufgrund ihrer Grundstofflastigkeit in der Industrieproduktion auch vom starken Rückgang der Rohstoffpreise negativ betroffen.

\subsection{KRISENBETROFFENHEIT IM VERGLEICH}

Die stärkste Rezession erlitten die baltischen Länder mit ihrer extrem neoliberalen Wirtschaftspolitik, ihren weit überdurchschnittlichen außenwirtschaftlichen Defiziten und ihrer schwachen Industriestruktur (vgl. Leitner 2010). Hier setzte die Krise teils schon Anfang 2008 ein. In Lettland und Estland ging das BIP bereits 2008 um 4,5 bzw. 3,6 \% zurück. 2009 erreichte der BIP-Rückgang in den Baltischen Ländern internationale Spitzenwerte: in Lettland 18,0 \%, in Litauen 15,0 \% und in Estland 14,1 \% (Pöschl 2010, S. 428, Tab. 1). Lettland erreichte damit gerade einmal das BIP-Niveau von 1990, und auch Litauen lag nur 8,5 \% darüber (ebd. S. 429, Tab. 2). Auch 2010 war nur eine (möglicherweise vorübergehende) Stabilisierung auf niedrigem Niveau - mit etwas höheren Wachstumsraten in Estland als in Lettland und Litauen - festzustellen (Eurostat 2011b). Die soziale Lage hat sich in den Baltischen Ländern dramatisch verschlechtert. Die Arbeitslosenrate hat sich während der Krise mehr als verdoppelt und erreichte im September 2010 in Lettland und Litauen etwas mehr als $18 \%$, in Estland $16,2 \%$. Bei den Jugendlichen war die Lage mit Arbeitslosenraten von gut einem Drittel in Lettland und Litauen sowie $28 \%$ in Estland noch trüber (Eurostat 2011a).

In der Ukraine ging das BIP 2009 ebenfalls um 15,1 \% zurück, womit gerade einmal 63 \% des BIP-Niveaus von 1990 erreicht wurden (Pöschl 2010, S. 428f., Tab. 1 und 2). In der Ukraine waren im Prinzip die gesamten 1990er Jahre durch wirtschaftlichen Rückgang und eine dramatische Verschlechterung der sozialen Lage gekennzeichnet. Der Aufschwung im letzten Jahrzehnt war stark kreditgetrieben, wobei die hohe Rohstofflastigkeit und Energieintensität als strukturelle Charakteristika der ukrainischen Wirtschaft bestehen blieben. Beide Elemente schlugen in der aktuellen Krise voll negativ durch. Die offene Arbeitslosigkeit stieg in der Ukraine nicht so stark an wie in den baltischen Ländern (Myant/Drahokoupil 2011, S. 336,
Tab. A 4), stattdessen wurden Löhne nur teilweise und/oder verspätet ausgezahlt.

Das Mittelfeld dieser Gruppe wurde von südosteuropäischen Ländern mit hoher Devisenverschuldung - Rumänien, Bulgarien, Kroatien und Montenegro sowie Ungarn gebildet. Hier ging das BIP 2009 zwischen 5,0 \% (Bulgarien) und 7,1\% (Rumänien) zurück - als Fortsetzung der rezessiven Tendenz im Jahr 2010 (Pöschl 2010, S. 428, Tab. 1; Eurostat 2011b). Hohe Auslands- und Devisenschulden stellten in diesen Ländern eine erhebliche Restriktion der Wirtschaftspolitik dar, die stark prozyklisch ausgerichtet war. Wenn das kroatische Wirtschaftsmagazin Banka mit Daten bis Oktober 2010 den weit höheren Stand der Arbeitslosigkeit in Kroatien (ca. 18 \%) und die dort weiter steigende Tendenz mit der deutlich niedrigeren Arbeitslosigkeit und abnehmender Tendenz in Rumänien und Bulgarien (7,1 \% bzw. 8,9 \%) kontrastiert (O.A. 2011a, S. 66), so ist zu berücksichtigen, dass sich im Krisenfall ein Teil der Bevölkerung in Ländern wie Rumänien und Bulgarien auf die Kleinlandwirtschaft zurückzieht. Ähnliche Reaktionen auf Krisensituationen gibt es auch in Serbien. Serbien und Bosnien-Herzegowina, wo die militärischen Konflikte besonders lange dauerten und der Verschuldungszyklus entsprechend verspätet einsetzte, wiesen 2009 mit einem BIP-Rückgang von $3 \%$ eine unterdurchschnittliche Rezession auf (Pöschl 2010, S. 428, Tab. 1). Für das produzierende Gewerbe in Serbien dürfte auch die Abwertung des Dinar die Krisenfolgen abgemildert haben.

Für die primär finanziarisierten Ökonomien gilt, dass deren Akkumulationsmodell der Vorkrisenzeit die Grundlage entzogen worden ist. Sie weisen in der Tendenz eine deutlich höhere Privatverschuldung, Außenverschuldung und noch größere Defizite im produktiven Bereich als die primär exportorientierten Ökonomien der Region auf. Sie haben tendenziell unter einer schärferen Rezession und dramatischeren sozialen Krisenfolgen als die exportorientierten Länder zu leiden.

\section{Wirtschaftspolitische Reaktionen auf die Krise}

Die Reaktionen auf die Krise sind in mehrfacher Hinsicht geteilt. Es zeigte sich, dass 
die primär exportorientierten Länder über größere wirtschaftspolitische Spielräume als die primär finanziarisierten Länder verfügten. Eine zweite Differenzierungslinie betraf EU-Mitgliedsländer und osteuropäische Staaten außerhalb der EU. Nur für Erstere hatten Entscheidungen der EU unmittelbare Konsequenzen. Die EU-Politik selbst war im Herbst 2008 zunächst vor allem auf die Krisensituationen der westeuropäischen Bankensektoren bezogen. Die Entscheidung, als systemrelevant eingeschätzte Banken unbedingt zu stützen, wurde in der Gruppe der EuroLänder zuzüglich Großbritannien gefasst, sodass die Mehrzahl der osteuropäischen EU-Länder hieran nicht beteiligt war. Mehr noch: Die besondere Situation der osteuropäischen Länder mit einem hohen Grad der informellen Euroisierung und der damit verbundenen Notwendigkeit, die Banken ggf. mit Kapitalspritzen in Devisen stützen zu müssen, war nicht Bestandteil dieser Entscheidung. Der entsprechende EU-Fonds wurde erst nach einigen Wochen aufgestockt - und dann auch gleich mit schärferen Bedingungen versehen (Wehr 2009, S. 76). Diese Konditionen kamen bei jenen EU-Ländern zur Anwendung, die Stützungskredite bei EU und IWF beantragten. Nicht mit speziellen Konditionen war allerdings 2009 die Einräumung einer Special Credit Line des IWF für Polen, die als Vorsichtsmaßnahme konzipiert war, verbunden. Außerhalb der EU konzentrierte sich hingegen die direkte äußere Intervention auf den IWF.

\subsection{REAKTIONEN DER EXPORT- ORIENTIERTEN LÄNDER}

Mit Ausnahme Weißrusslands reagierten die Regierungen der Länder mit primär exportorientierter Entwicklung - Polen, Slowakei, Slowenien, Tschechische Republik - formal autonom auf die Krise. In einer ersten Phase lancierten sie einige gering dimensionierte anti-zyklische Maßnahmen wie eine Verschrottungsprämie bei Autos oder Subventionen für die Wärmedämmung. Auch eine größere Flexibilität bei der Kurzarbeit wurde für Länder wie die Slowakei oder Polen eingeführt (Becker 2010, S. 528f.). Abgesehen von einer Erhöhung der Mehrwertsteuer hat die polnische Regierung dieses Politikmuster seitdem fortgesetzt, wofür sie inzwischen von Seiten von Rating-Agenturen verstärkt kritisiert wird (O.A. 2010). In der Tschechi- schen Republik und der Slowakei waren anti-zyklische Politikmaßnahmen und die steigende Staatsverschuldung ein zentrales Wahlkampfthema im Frühjahr 2010.

Trotz des geringen Standes der Staatsverschuldung-im Jahr 200935,4\% des BIP in beiden Ländern (Schreiner et al. 2010, S. 7, Tab.4 und S. 33, Tab. 7) - malten liberale Medien bereits im Jahr 2009 das Horrorbild eines drohenden Staatsbankrotts an die Wand (z.B. Sachr/Švehlar 2009). In der Slowakei machten die Rechtsparteien ihre Ablehnung der slowakischen Beteiligung am Stützungspaket der Euro-Zone für Griechenland zum Wahlkampfthema. Damit hatten sie Erfolg. Für den liberalen slowakischen Publizisten Martin Šimečka (2010, S. 15) hat die Frage des Griechenland-Pakets in der Slowakei möglicherweise sogar den Ausschlag zugunsten der Rechtsparteien gegeben. Der Wahlsieg der Rechten führte in beiden Ländern zu einem Einschwenken auf eine pro-zyklische Sparpolitik. In beiden Fällen sind Kürzungen der öffentlichen Personalausgaben und der Sozialleistungen Kern dieser Sparpolitik. Weiterhin ist in beiden Ländern eine Liberalisierung des Arbeitsrechts - also ein Abbau von Rechten der abhängig Beschäftigten - in Vorbereitung. Unter der Flagge der Krisenbekämpfung geht es um eine strategisch angelegte Radikalisierung neoliberaler Politikmuster. Das gilt vor allem für die Tschechische Republik, wo selbige bislang nicht so ausgeprägt sind wie in der Slowakei, Ungarn oder Polen. In der Tschechischen Republik gab es gegen Ende 2010 eine Großdemonstration sowie einen Streiktag im öffentlichen Dienst gegen die Sparpolitik (Becker 2010, S. 530). Auch die slowakischen Gewerkschaften kündigten Proteste gegen die Veränderung des Arbeitsrechtes an.

In Polen und der Tschechischen Republik hat sich angesichts der Krise eine stärker abwartende Haltung zur EuroEinführung durchgesetzt (Becker 2010, S. 530f.). In der Slowakei sind die Folgen des Beitritts zur Euro-Zone im Hinblick auf die Beteiligung an den Stützungspaketen weiterhin ein umstrittenes Thema (vgl. z.B. Szomolányi 2010; Marcinčin 2010). Der franko-tschechische Intellektuelle Jacques Rupnik fühlt sich angesichts der Töne „Wir werden nicht für die faulen Griechen zahlen", wie sie in der Slowakei aktuell laut werden, an tschechische Positionierungen kurz vor der Trennung von der Slowakei vor 20 Jahren erinnert. Damals habe es analog geheißen, „warum haben wir für die Slowaken zu zahlen“" (Interview durch Balážová 2010, S. 22). Mithin deuten sich Veränderungen in der Haltung der führenden politischen Kräfte im Hinblick auf die EU-Integration an.

Im Gegensatz zu den exportorientierten neuen EU-Mitgliedsländern beantragte die weißrussische Regierung im Januar 2009 ein 15-monatiges Programm beim IWF. Zu diesem Zeitpunkt hatte die weißrussische Regierung - entsprechend der IWF-Konzpetionen - dem Bankensektor bereits eine Kapitalspritze zukommen lassen, um Verluste aufgrund der Krise und der partiellen Abwertung der weißrussischen Währung auszugleichen. Die Kapitalspritze für den überwiegend staatlichen Bankensektor in Weißrussland war vergleichsweise deutlich kleiner als für die privatisierten Bankensektoren in der Ukraine und Kasachstan (Barisitz et al. 2010, S. 63). Im Gegensatz zu den stärker informell euroisierten Staaten Osteuropas befürwortete der IWF im Fall Weißrusslands eine begrenzte Währungsabwertung, die zu einer Besserung der Leistungsbilanz beitragen sollte (IMF/Republic of Belarus 2009, S. 16f.). Das heißt, dass im Fall von Weißrussland der Entwicklung der Leistungsbilanz größere Aufmerksamkeit als bei den anderen osteuropäischen Ländern beigemessen wurde und der Anpassungsdruck - im Gegensatz zu den anderen IWFProgrammen - nicht weitgehend auf den Löhnen lastete. Dies dürfte im Interesse der autoritären Regierung Lukašenko gelegen haben, deren Legitimität vor allem auf der Verbesserung der sozialen Lage fußt. Die Fiskalpolitik wurde für 2009 auch für Weißrussland restriktiv ausgerichtet, wenn auch nicht im selben Ausmaß wie in anderen osteuropäischen Ländern. Der IWF forderte zudem Liberalisierungsmaßnahmen in der stark etatistisch regulierten weißrussischen Ökonomie (IMF/Republic of Belarus 2009, S. 20ff.). Diese eher orthodoxen Maßnahmen komplementierte die weißrussische Regierung durch heterodoxe Maßnahmen wie verschärfte Devisenkontrollen (Barisitz et al. 2010, S. 66, 76). Die Konditionalität des IWF scheint im weißrussischen Fall relativ gemäßigt. Die weißrussische Regierung hätte grundsätzlich auch die Alternative gehabt, Russland um finanzielle Unterstützung zu ersuchen. In dieser Phase versuchten die westlichen Länder, die Regierung Lukašenko stärker von Moskau abzukoppeln. Das scheint sich 
in der IWF-Politik widerzuspiegeln. Nach der heftigen Repression nach den Präsidentschaftswahlen in Weißrussland Ende 2010 ist das Tauwetter zwischen Minsk und Brüssel wieder vorbei. In der Schaukelpolitik Lukašenkos ist wieder eher Moskau an der Reihe, was sich schon im Vorfeld der Wahlen abzeichnete (vgl. Kłysiński 2011, S. VII).

\subsection{REAKTIONEN DER LÄNDER MIT FINANZIARISIERTEN AKKUMULA- TIONSREGIME}

In den Ländern mit einem primär finanziarisierten Akkumulationsregime ist die Politik von Anfang an stark pro-zyklisch ausgelegt - und das unabhängig davon, ob Abkommen mit dem IWF bzw. IWF und EU getroffen wurden oder nicht. Die Kernausrichtung der Politik ist es, den Wechselkurs um jeden Preis stabil zu halten. Dies liegt einerseits im Interesse der westlichen Banken und findet andererseits auch die Unterstützung der in Devisen verschuldeten Mittelschichten. Für die westeuropäischen Banken würden Währungsabwertungen in Osteuropa eine Abwertung ihrer Aktiva in der Region bedeuten, was sie vermeiden möchten. Außerdem würden durch eine Währungsabwertung die Fremdwährungsschuldner in große Probleme geraten. Daher genießt die Wechselkursstabilisierung auch bei den Mittelschichten breite Unterstützung (Becker 2010, S. 532f.). In den Baltischen Ländern ist die Orientierung auf die EuroEinführung ungebrochen und erfolgte in Estland - trotz der enormen Leistungsbilanzdefizite in den Vorjahren - auch tatsächlich Anfang 2011.

Um die Leistungsbilanzdefizite zu senken, wurde daher statt auf eine Abwertung auf eine deflationär ausgerichtete extreme Sparpolitik gesetzt, bei der Lohnsenkungen im öffentlichen Dienst und Abbau von Sozialleistungen eine zentrale Rolle spielen. So sollen die Binnen- und damit die Importnachfrage gesenkt werden. Diese Maßnahmen haben z.T. zu sozialen Protesten, vor allem in Rumänien, und vereinzelt auch zu Streiks im öffentlichen Dienst geführt. Die Proteste haben bislang nicht die Stärke erreicht, dass dieses Politikmuster grundsätzlich infrage gestellt würde. Das unterscheidet Osteuropa bislang von den damals teil-dollarisierten Staaten des Cono Sur in Südamerika, die angesichts der schweren Krise und dem Scheitern ih- rer deflationär ausgerichteten Sparpolitik Anfang 2002 die Währung abwerteten und im Fall Argentinien auch eine substanzielle Reduktion der Auslandsschuld durchsetzten (Becker 2008, S. 168ff.).

Dem Grundmuster von Währungsstabilisierung und deflationär orientierter Sparpolitik gehorchen sowohl die Stützungsprogramme, die von IWF und Europäischer Kommission für die EUMitgliedsstaaten Ungarn, Lettland und Rumänien akkordiert wurden, als auch die IWF-Programme für die Ukraine, Serbien und Bosnien-Herzegowina (Becker 2009, S. 102ff.; Becker 2010, S. 533ff.; Galgóczi 2009, S. 5f.; Drezgić 2010, S. 215ff.). Makedonien wurde Anfang 2011 Zugang zu einer erst 2010 geschaffenen Precautionary Credit Line, einer weicheren Option als den üblichen Stand-By-Abkommen, eingeräumt. Auch bei dieser Kreditlinie stand die Wahrung der Parität im Vordergrund (O.A. 2011b; IMF 2011).

Die Europäische Kommission setzte im Interesse der westeuropäischen Banken noch eindeutiger als der IWF auf Stabilisierung der Währungsparität - dies wurde zumindest im Fall Lettlands erkennbar (Leitner 2010, S. 50; Wehr 2010, S. 45, 105). Ähnlich kompromisslos zeigte sie sich in der Austeritätspolitik. Im Fall Lettlands und Rumäniens sind die Kürzungen im Budget und bei den Gehältern der öffentlich Bediensteten besonders hoch ausgefallen (Becker 2010, S. 533). Es lässt sich bei den Abkommen eine eindeutige Tendenz zu Lohnkürzungen und Abbau von Sozialleistungen, speziell bei den Pensionen, feststellen. Diese Maßnahmen in den Peripherieländern werden auch Rückwirkungen auf die Kernländer der EU haben. Von budgetären Sparmaßnahmen waren Re-Kapitalisierungsmaßnahmen für den Bankensektor ausgenommen. Bereits Mitte 2009 - noch lange vor Abschluss der Sanierungsmaßnahmen - beliefen sich in der Ukraine die staatlichen Stützungskosten für die Banken auf $5 \%$ des BIP (Barisitz et al. 2010, S. 59). Die Ukraine verband die orthodoxe Politik mit einzelnen heterodoxen Maßnahmen, beispielsweise verschärften Devisenkontrollen. Im Vorfeld der Präsidentschaftswahlen erhöhte das ukrainische Parlament Mindestlöhne und Pensionen, womit das IWF-Abkommen vom Kurs abkam (Barisitz et al. 2010, S. 11). Die neue ukrainische Regierung, die sich stark auf die Schwerindustrie im Osten des Landes stützt und stärker auf gute Beziehungen mit Russland setzt, unterschrieb einerseits im Sommer 2010 ein neues Abkommen mit dem IWF (Górska 2010, S. 17f.) und handelte andererseits mit Russland ein neues Gasabkommen aus. Dieses bringt als Gegenleistung für die Verlängerung von Stützpunktverträgen im Schwarzen Meer deutliche Erleichterungen für die sehr energieintensive Schwerindustrie und wirkt sich günstig auf die ukrainische Handelsbilanz aus (Marušiak 2010, S. 47). Es zeigt sich also, dass nicht nur Weißrussland, sondern auch die Ukraine als Länder der äußeren europäischen Peripherie über mehr Handlungsspielräume als die osteuropäischen EU-Mitgliedsländer verfügen.

Unter bestimmten Umständen haben allerdings auch die Regierungen von hoch verschuldeten EU-Mitgliedsländern bestimmte Spielräume. Das zeigt der ungarische Fall, wo die neue Fidesz-Regierung 2010/2011 mit der Einführung von Sondersteuern für die Banken und andere hochkonzentrierte Wirtschaftssektoren mit starker Präsenz westlicher Konzerne sowie einer Rekanalisierung der Pensionsbeiträge in das öffentlichen Pensionssystem sich Raum für Steuererleichterungen für ihre Wählerklientel schaffte und damit IWF, Europäische Kommission sowie westliche Unternehmen verärgerte (Becker 2011).

Die extreme Sparpolitik hat tatsächlich zu einer deutlichen Verbesserung bei den Leistungsbilanzdefiziten und damit einer Minderung des laufenden externen Finanzierungsbedarfs geführt. Allerdings hat sich die Verschuldungssituation weiter verschärft. Die Kreditausfälle nehmen weiter zu (OeNB 2010, S. 24, Abb. 13). Aufgrund der starken Rezession ist sowohl die private als auch die öffentliche Schuldenlast weiter gestiegen - beispielsweise die Last der Auslandsschuld allein zwischen 2008 und dem 2. Quartal 2009 in Lettland von 128 auf $156 \%$ des BIP, in Rumänien von 50 auf $68 \%$ des BIP, in Ungarn von 78 auf $95 \%$ und in Kroatien von 83 auf $95 \%$ des BIP (Hrvatska Narodna Banka 2010, S. 12, Tab. 3). Die produktiven Strukturen werden durch die rezessiv angelegte Politik weiter geschwächt. Damit werden die Defizite im produktiven Bereich zementiert. Speziell in den baltischen und den südosteuropäischen Ländern ist unklar, wie mit derart schwachen produktiven Strukturen auf längere Sicht der Schuldendienst aufgebracht werden soll. Damit ist die deflationär angelegte Wirtschaftspolitik mit erheblichen inneren Widersprüchen 
verbunden. Die Entwicklungsperspektiven der bislang auf eine Finanziarisierung ausgerichteten Ökonomien Osteuropas sind als katastrophal und sehr instabil einzuschätzen.

\section{Schlussfolgerungen}

In der Tendenz hatte die Krise in den primär finanziarisierten Ökonomien Osteuropas stärkere Auswirkungen als in den eher exportorientierten Ländern. Besonders schwer waren die neo-liberalen Musterländer des Baltikums mit ihrer starken Beschleunigung der Verschuldung und den extremen Leistungsbilanzdefiziten betroffen. Auch in der Anti-Krisen-Politik sind Differenzierungen zwischen den beiden Akkumulationsmodellen festzustellen. Insgesamt ist die Wirtschaftspolitik - im Interesse der westlichen Banken und der in Devisen verschuldeten Mittelschicht - in den bislang primär finanziarisierten Ökonomien noch restriktiver ausgerichtet als in den primär exportorientierten Ökonomien.
Einen Einfluss auf die wirtschaftspolitische Ausrichtung hatte allerdings auch die innenpolitische Konstellation. So nutzten neu gewählte Rechtsregierungen in der Tschechischen Republik und der Slowakei die Krise als Vorwand zu einer vertieften neoliberalen Politik. Erkennbar ist auch, dass die wirtschaftspolitische Autonomie zumindest in Teilen der äußeren Peripherie - Weißrussland und der Ukraine - höher war als bei den osteuropäischen EU-Mitgliedsländern, die sich zum Teil einem sehr harten Druck vonseiten der Europäischen Kommission und des IWF ausgesetzt sahen.

\section{LITERATUR}

Astrov, V./Pöschl/J. (2009): MOEL im Sog der Krise, in: WifoMonatsberichte 82 (5), S. 347-359

Balážová, D. (2010): Jacques Rupnik: Pílite si pod sebou konár, in : Zahraničná politika 14 (5), S. 21-22

Baletić, Z./Grqić, M./Lasić, V./Lovrinović, I./Marendić, B./

Radošević, D./Zdunić, S. (2010): Prosudba Memoranduma o ekonomskoj a financijskoj politici hrvatske Vlade i HNB-a upućenog MMF-u., in: Radošević, D. (Hrsg.): Kriza i ekonomska politika. Politika i ekonomija razvoja Hrvatske, Zagreb, S. 17-64

Barisitz, St. (2009): The Belarussian Banking System, in: Heinrich, H. G./Lobova, L. (Hrsg.): Belarus: External Pressure, Internal Change, Frankfurt/M. u.a.O., S. 217-236

Barisitz, St./Holzhacker, H./Lytvyn, O./Sabyrova, L. (2010):

Crisis Response Policies in Russia, Ukraine, Kazakhstan and Belarus Stock-Taking and Comparative Assessment, in: Oesterreichische Nationalbank (OeNB): Focus on European Economic Integration (4), S. 48-77 Becker, J. (2008): Zyklen von Finanzialisierung, Verschuldung und Exportorientierung. Argentinien, Brasilien und Uruguay, in: Küblböck, K./Staritz, C. (Hrsg.): Asienkrise: Lektionen gelernt? Finanzmärkte und Entwicklung, Hamburg, S. 159-174

Becker, J. (2009): Osteuropa in der Finanzkrise: Ein neues Argentinien? in: Blätter für deutsche und internationale Politik 54 (6), S. 97-105 Becker, J. (2010): Krisenmuster und Anti-Krisen-Politiken in Osteuropa, in: Wirtschaft und Gesellschaft 36 (4), S. 517-540

Becker, J. (2011): Die nationalistische Variante des Neoliberalismus, in: Informationsbrief Weltwirtschaft \& Entwicklung vom 3.1., www.weltwirtschaft-und-entwicklung.org abgerufen: 4.1.2011 Burić, I. (2010): Nacija zaduženih. Od komunističkog pakla do potrošačkog kapitalizma, Zagreb
Drezgić, S. (2010): Fiskalna politika europskih tranzicijskih zemalja u uvjetima recesije, in: Radošević, D. (Hrsg.): Kriza i ekonomska politika. Politika i ekonomija razvoja Hrvatske, Zagreb, S. 205-224

Dutrénot, G./Sand-Zantman, A. (2010): Après la crise? Les politiques économiques dans le monde, Paris

Dvorsky, S./Schreiber, Th./Stix, H. (2010): Real Effects of Crisis Have Reached CESEE Households: Euro Survey Shows Dampened Savings and Changes in Borrowing Behavior, in: Oesterreichische Nationalbank (OeNB): Focus on European Integration (2), S. 79-90

Eurostat (2011a): Arbeitslosenquote des Euroraums bei 10,1 \%, Eurostat pressemitteilung euroindikatoren (5) vom 7. Januar Eurostat (2011b): BIP im Euroraum um 0,3 \% und in der EU-27 um $0,5 \%$ gestiegen. Eurostat pressemitteilung euroindikatoren 6 vom 7. Januar 2011

Frangakis, M. (2009): Europe's financial system under pressure, in: Grahl, J. (Hrsg.): Global Finance and Social Europe, Cheltenham, S. $53-90$

Galgóczi, B. (2009): Central and Eastern Europe Five Years After: From „Emerging Europe" to "Submerging Europe"? ETUI Policy Brief (4) Górska, A. (2010): Ukraina, in: Nowa Europa Wschodnia 5 (13), S. $16-18$

Hrvatska Narodna Banka (2010): Financijska stabilnost (5), Zagreb International Monetary Fund (IMF/IWF)/Republic of Belarus (2009): Request for Stand-By Agreement, www.imf.org/external/pubs/cat/ longres.aspx?sk=22853.0 (abgerufen 28.1.2011)

International Monetary Fund (IMF/IWF) (2011): IMF Executive Board Approves $€ 475.6$ million Arrangement for Macedonia under the Precautionary Credit Line, Press Release 11714, January 19, www.imf.org/ external/np/sec/pr/2011/pr1114.htm (abgerufen 29.1.2011) 
Kłysiński, K. (2011): Gra czy przyjaźń, in: Tygodnik Powszechny, vom 16.1., S. VII

Kovačević, M. (2009): Osnovi elementi ekonomsko-finansijskih odnosa Srbije sa inostranstvom, in: Stojić Karanović, E./Janković, S. (Hrsg.): Elementi strategije spoljne politike Srbije, Belgrad, S. 265-275

Leitner, S. (2010): Baltikum: Ein neoliberales Transformationsmodell fordert seine Opfer, in: Kurswechsel (1), S. 47-53

Ludwig, M. (2011): Motivsuche im Dunkel des Lukaschenka-Regimes, in: Frankfurter Allgemeine vom 13.4, S. 3

Marcinčin, A. (2010): Pomôct' je viac ako poučuvat', in: Zahraničná politika 14 (5), S. 27-28

Marn, U. (2010): Grabinci na udaru, in: Mladina vom 19.3., S. 36-40

Marušiak, J. (2010): Ukrajina sa obracia na Východ,

in: Pravda vom 24.4., S. 47

Marzęda, K. (2009): Pozycja ekonomiczna Białorusi, in: Topolski, I.

(Hrsg.): Białoruś w stosunkach mięzynarodowych, Lublin, S. 137-157

Mazzochi, R. (2010): Europe nella crisi globale, in: Gualteri, R./

Rhi-Sausi, J. (Hrsg.): Hub globale, trincea o pantano? Il futuro del Mediterraneo e il ruolo dell'Europa, Rapporto 2010 sull'integrazione europea. Bologna, S. 249-284

Morvay, K. u. Autorenkollektiv (2010): Hospodarský vývoj Slovenska v roku 2009, Bratislava

Murahemi-Vukas, S. (2009): Intervju: Danilo Tirk, predsednik Slovenije: Podržavamo brži ulazak Srbije u EU, in: NIN vom 31.12., S. 66-68 Myant, M./Drahokoupil, J.(2011): Transition Economies: Political Economy in Russia, Eastern Europe, and Central Asia, Hoboken

O.A. (2010): Fitch: Polska potrzebuje poważnych reform, by utrzymać rating, in: Gazeta Wyborcza vom 25.11., www.gazta.biz/ biznes/20290202,101562,8717236,Fitch__Polska_potrzebuje_ powaznych-reform__by-utrzymac.html (28.1.2011)

O.A. (2011a): Dobre vijesti iz Rumunjske i Bugarske, in: Banka 17 (1), S. 66

O.A. (2011b): Kredit za svaki slučaj, in: Danas vom 21.1., S. 9

Oesterreichische Nationalbank (OeNB) (2010): Fragile Erholung nach der Krise, in: OeNB: Finanzmarktstabilitätsbericht 20, Wien, S. 9-24
Okáli, I. u. Autorenkollektiv (2008): Hospodarský vývoj Slovenska v roku 2007, Bratislava

Pann, J./Seliger, R./Übeleis, J. (2010): Foreign Currency Lending in Eastern and Southeastern Europe: the Case of Austrian Banks, in: OeNB: Finanzmarktstabilitätsbericht 20, Wien, S. 60-80

Petković, D./Nikolić, G. (2009): Geopolitika savremene Ukrajine, Belgrad

Poeschl, J. (2010): Erholungstendenzen in den MOEL, in: Wifo-Monatsberichte 83 (5), S. 427-443

Sachr, T./Švehla, M. (2009): Zachraní nás premier Škrt, in: Respekt vom 17.8., S. 16-19

Schreiner, J./Barisitz, St./Eller, M./Fidrmuc, J./Gardó, S./ Hildebrandt, A./Lahnsteiner, M./Reiniger, Th./Walko, Th./ Wörz, J. (2010): Developments in Selected CESEE Countries: Multispeed Recovery in CESEE, in: OeNB: Focus on European Economic Integration (4), S. 6-45

Šimečka, M. M. (2010): V bahnĕ populismu, in: Repekt vom 19.7., S. $14-15$

Szomolányi, S. (2010): Grécka kríza a slovenské nie, in: Zahraničná politik 14 (5), S. 23-24

Urbinati, E. (2010): Slovenia, in: Privitera, F. (Hrsg.): Guida ai paesi dell'Europa centrale orientale e balcanica. Anuario politico-economico 2009, Bologna, S. 325-334

Wehr, A. (2009): Währung mit eingebauter Sozialdemontage. Der Euro und die Krise, in: Pfeiffer, H. (Hrsg.): Land in Sicht? Die Krise, die Aussichten und die Linke, Köln, S. 69-80

Wehr, A. (2010): Griechenland, die Krise und der Euro, Köln

Workie, M. T. u. Autorenkollektiv (2009): Vývoj a perspektivy svetovej ekonomiky. Globálna finančná a hospodárska kríza. Príčiny - náklady východiská, Bratislava

Žold', O. (2010): Kredit MWF dlja Ukraïni, in Ukraïnskij Žurnal (9-10), S. $44-45$ 